\title{
ACTIVITY TRACERS
}

\author{
KLAUS G. STRASSMEIER \\ Institut für Astronomie, Universität Wien, \\ Türkenschanzstraße 17, A-1180 Wien, Austria
}

\begin{abstract}
The vast variety of solar-like phenomena on other late-type stars, so-called activity tracers, provide an important tool for studying the structure of active stellar atmospheres and their connection to the stellar interior via strong magnetic fields. These "chromospherically active" stars include single and binary stars as well as pre- and postmain sequence objects and have rapid rotation and deep convective layers in common. They serve as astrophysical laboratories to study the vast phenomenology of activity tracers: starspots, plages, flares, prominences, which might be seen as enhanced analogs of solar activity and could be spatially resolved due to rotationally modulated indicators. In this paper we review the current observational material and discuss its impact on our knowledge of "active" atmospheres, especially in the context of stellar rotation.
\end{abstract}

\section{ACTIVITY TRACERS THROUGHOUT THE HR DIAGRAM}

Convective envelope stars: the $F, G$, and $K$ stars

It is generally thought that stellar magnetic activity is determined from the interaction of stellar rotation and convection through a dynamo mechanism. The relation between various surface activity indicators and stellar (surface) rotation has been amply demonstrated (eg. Hartmann \& Noyes 1987, Liebert \& Probst 1987 , and references therein). Similar rotation-activity relations for evolved stars also exist but are less well defined due to a much larger range of internal constitutions for a given spectral type (Basri et al. 1985, Simon \& Fekel 1987, Young et al. 1989, Stepien 1989, Strassmeier et al. 1990a, Pasquini et al. 1990, Böhm-Vitense 1992). The appearance of large scale activity tracers in stellar atmospheres is only obvious for the most active stars, e.g., for components in close binaries (RS CVn's, Algols, W UMa's), for some very young stars (e.g. AB Dor, LQ Hya), and for anomalously rapidly rotating single giants (FK Comae's, and others). Figure 1 shows the position of such active stars in the H-R diagram compared to the granulation and coronal boundaries (adapted from Appourchaux et al. 1991).

Recent reviews concerning various subsets of stellar activity may be found in (chronological order): Linsky (1992) "Relations between activity indicators", Schmidt (1992) "Sunspots", Eaton (1992) "Starspot models", Strassmeier (1992) "Starspot photometry", Pallavicini (1992) "Stellar flares", Hall (1991a) "Longterm photometry", Gray (1991) "Rotation of evolved stars", Saar (1991) "Magnetic field measurements", Bruning (1991) "Mean magnetic field", Vilhu (1990) "Stellar activity", Ramsey (1990) "Optical and UV spectra of RS CVn's", Catalano (1990) "Flares in RS CVn binaries", a.o.. 
Radiative envelope stars: the A and B stars

The Herbig Ae/Be stars

These stars are believed to be pre-main sequence objects of intermediate mass (Finkenzeller \& Mundt 1984). Extensive chromospheres and stellar winds were detected for $\mathrm{AB}$ Aur (A0Ve; Catala 1988), who also estimated the radiative losses from the chromosphere of $\mathrm{AB}$ Aur to be about $6 \%$ of the star's bolometric luminosity, as compared to $10^{-3}$ for most RS CVn's. A0 stars do not have convective envelopes and thus need some other "source" for providing these stars with a chromosphere. It is usually believed that magnetic fields play the dominant role for transporting the energy to greater heights in the atmosphere, but no direct detection of a magnetic field has yet been reported. It has been proposed that a magnetic field is also the cause of the stream structure in the wind of AB Aur (Praderie et al. 1986). Indirect evidence for the existence of magnetic fields comes from the observation of periodic variations of chromospheric resonance lines such as $\mathrm{Mg} I \mathrm{k}$ and $\mathrm{Ca} I \mathrm{~K}$, which are thought to be formed in (corotating) stream winds (cf. Catala et al. 1991).

The chemically peculiar Ap stars

The transition between solar-type magnetic fields, i.e. isolated flux tubes embedded in non-magnetic regions, and Ap or Bp-type fields, i.e. poloidal fields covering the entire atmosphere with an usually dipolar structure, seems to occur around $T_{\text {eff }} \approx 7000 \mathrm{~K}$ or $\approx$ F0 (cf. Landstreet 1991 ), about where deep convective envelopes begin to develop. Wolff et al. (1986) finds the onset of chromospheric activity at $B-V=0.28$ or $\approx 7300 \mathrm{~K}$ as deduced mainly from C IV $1549 \AA$ line fluxes. The relationship between CIV $1549 \AA$ flux and photospheric magnetic fields over quiet and over active solar regions was studied by Schrijver (1990) who found further evidence that a small fraction of CIV flux (the basal flux defined by Schrijver 1987) is not related to magnetic fields. Generally, no chromospheric emission is seen from Ap stars with a few exceptions: C I emission from the $\delta$ Scuti variable $\Theta$ Tuc (A7IV, $B-V=0.23$ ) and possibly HR 4086 (A8V, $B-V=0.25$ ), but definitively in 83 Tau (F0V, $B-V=0.26$ ). For a summary see Simon \& Landsmann (1991). No directly measured magnetic fields were found in non-Ap stars earlier than G0. Extensive observations of Ap stars have shown that fields are likely associated with an inhomogeneous surface distribution of certain chemical elements. Their detection through line-profile variations and consequent Doppler imaging provides an activity tracer for Ap stars (Hatzes et al. 1989, Rice \& Wehlau 1990, 1991, Hatzes 1991a,b). Recently, magnetic fields were spatially resolved on the Ap star $\epsilon \mathrm{UMa}$ by means of the Zeeman Doppler imaging technique (Donati et al. 1990) and show that the pole of the ring-like structure of excess iron and chromium abundance is consistent with the location of the pole of the magnetic field. It seems that certain surface structures are, within their uncertainties (see Rice 1991), indicative of magnetic fields on these type of stars. This seperation of elements has also been searched for on the Sun (cf. Zirin 1988). One might expect the heavier ions to sink more rapidly than the lighter. However, mixing through turbulence appears to 
restore a certain balance. Observations are not yet conclusive whether there is an underabundance of elements with a high first ionization potential.

\section{STELLAR PLAGES}

\section{Integrated flux measurements}

While the solar photosphere appears chaotic, the bulk of the magnetic field occurs in a limited range of structures: in active regions (plages, spots, fibrils), in unipolar regions, and ephemeral active regions. The existence of bright, chromospheric patches on stars other than the Sun was probably best demonstrated by the Ca II H \& K emission survey at Mt. Wilson observatory (e.g. Wilson 1978, Baliunas et al. 1983, Baliunas \& Vaughan 1985) but also by early work with IUE (e.g. Marstad et al. 1982). First evidence of correlations between enhanced chromospheric and transition region emissions and the visibility of starspots was presented by Baliunas \& Dupree (1982) for $\lambda$ And and by Baliunas (1988) for $\delta$ CrB. From such correlations, it seems clear now that stellar plages exist and are warmer than the underlying photosphere (cf. Mathys \& Solanki 1989), but how warm remains to be determined.

But life is not that easy that we could conclude that stellar plages are generally associated with spots. They might be at some times, but not necessarily. This is nicely demonstrated by work that has been done with the IUE satellite on active stars like II Peg, AR Lac, BY Dra, V711 Tau, AU Mic, a.o.. Take for example II Peg: Rodonó et al. (1987) observed a clear enhancement of chromospheric and transition-region (TR) lines in anticorrelation with simultaneous $V$ and $V-I$ photometry in 1981, while two years later no modulation at all was seen (Andrews et al. 1988) although the star showed a $V$ amplitude of 0.11 mag. In 1986, clear evidence for rotational modulation in the $\mathrm{Mg}$ II lines was again present, however this time in the sense that higher flux was observed when the major spot concentration was out of view (Doyle et al. 1989). Strangely enough, higher temperature lines like $\mathrm{CII}$ and $\mathrm{CIV}$ (but also $\mathrm{O}_{\mathrm{I}}$ ) did show a modulation in anticorrelation with the simultaneous Fine-Error-Sensor light curve. At this time II Peg showed a full peak-to-peak amplitude of 0.5 mag (Byrne 1986, Doyle et al. 1988) which was at that time the largest amplitude ever measured for a spotted star.

Similar plage-spot correlation studies were carried out also for other stars with divergent results: no clear evidence was found for V711 Tau nor AR Lac (Rodonó et al. 1987). For HK Lac, MgII was stronger at times of spot visibility (K. Olah, priv. comm.), for IM Peg chromospheric lines (UV lines and Ca II infrared-triplet lines) were modulated by $25 \%$ while transition-region lines were modulated by 50 $\%$ (Huenemoerder et al. 1990) both in phase with contemporaneous photometry. The lesson seems clear: strict solar analogy is only valid for the less active stars while "overactive" stars like the RS CVn's cannot be viewed simply as scaled-up versions of solar-type behavior. 
Spectral imaging of stellar chromospheres

From ultraviolet lines

To go beyond simple correlations between activity indicators one must spatially resolve the surface features of interest. Non-uniformities in the chromospheric brightness distribution will produce emission components that vary due to the Doppler shift produced by the star's rotation. This led to a technique called spectral imaging (cf. Neff 1992). The longitudinal position and extent of a particular stellar plage are determined by the radial velocity of the line centroid and the line width, respectively. The latitudinal position and extent are determined by the radial velocity amplitude and the total time required by the plage to cross the stellar disk. So far, this technique has been applied to only a mere handful of stars most of them not even published yet, e.g. EI Eri and HD 199178 in Neff (1991) or HD 32918 in Vilhu et al. (1991). The only star with published maps is the double-lined, eclipsing RS CVn binary AR Lac (Walter et al. 1987, Neff et al. 1989, Pagano et al. 1992).

Using a series of high-resolution Mg II k line spectra, Neff et al. (1989) derived the first "image" of the chromosphere of AR Lac (G2IV+K0IV). They identified three distinct plages on the $\mathrm{K}$ star and a flare on the $\mathrm{G}$ star (Fig. 2; adapted from Neff et al. 1989). The trailing hemisphere of the $\mathrm{K}$ star was globally $40 \%$ brighter than the leading hemisphere. The $\mathrm{G}$ star also showed some brightness variations (by a factor of three) which the authors attributed to a large inactive region. Note that there is no direct analog for that on the Sun. A comparison with similar results from 1983 (two years earlier) suggests that the individual plages are long lived. A follow-up study for the years 1987 and 1989, also including broad-band photometry, was presented by Pagano et al. (1992). The three plages seen first in 1983 were still present but located at different longitudes (Fig. 3; adapted from Pagano et al. 1992). They had drifted at a speed of $-14^{\circ} \pm 10^{\circ}$ per year. Although no conclusive evidence of systematic spatial correlations with spots was found, their joint migration direction and rate suggests a common cause: magnetic fields anchored somewhere in the deep convection zone.

\section{From optical lines}

Stellar plages are also observable from optical wavelengths, mostly from the modulation of Ca II lines ( $\mathrm{H}, \mathrm{K}, 8498 \AA, 8542 \AA$, and $8662 \AA$ ). Once again the Sun is a useful guide. A comparison of spatially resolved solar quiet-region and plage profiles for the five CaII lines was presented by Shine \& Linsky (1972). Solar plages fill in the line cores, and the stronger the plage emission the more intense it is in the line wings, a fact mostly neglected in stellar work. The ratio of the core intensities of $\mathrm{Ca} I 1 \mathrm{H}$ to $\mathrm{K}$ increases from about 1.0 in quiet regions to about 1.1 in plages and might be a useful diagnostic for stellar plages. However, strong $\mathrm{H}$ and $\mathrm{K}$ emission is also seen from sunspots (Mattig \& Kneer 1978), even when only light from the umbral regions is analyzed, and yields an $H$ to $K$ emission peak ratio of 1.7 (Shine \& Linsky 1972). So the detection of Ca II linecore ratios greater than 1.0 does not necessarily imply that plages are the sole 
contributors, although they certainly contribute more light. This behavior is still to be investigated for other stars, but high-resolution line profiles $\left(\lambda / \Delta \lambda \approx 10^{5}\right)$ are required (e.g. Pasquini et al. 1988). Probably the most promising technique to study stellar plages is to analyze rotationally modulated fluxes combined with simultaneous spectral imaging.

\section{Magnetic field measurements}

It seems clear that the bulk of magnetic fields detected on cool stars must reside in bright regions: in plages, facular areas, and the network. Starspots contribute so little light to the Zeeman line profile at optical wavelengths that the magnetic field would be hard to detect (Saar 1988, Donati et al. 1992; see also the review by Saar 1991).

A particularly interesting star is the K5V BY Dra binary V833 Tau. No rotational modulation of chromospheric activity indicators was detected despite the high level of chromospheric emissions and magnetic field coverage $(B=2600 \mathrm{G}$, $f_{B}=0.5$, Saar $1991 ; f_{\text {spot }} \approx 0.2$, Saar \& Neff 1990). The star's low inclination of $10-23^{\circ}$ (Tokovinin 1990) suggest that the activity must reside at or near the visible pole, confirming the existence of chromospherically bright regions at very high latitudes. Dark photospheric features were already claimed to exist at or near the rotation pole on V833 Tau (Olah \& Pettersen 1991) and on other stars (e.g. Vogt 1988, Strassmeier 1990, Dempsey et al. 1992). Again, a phenomenon not seen on the Sun. Just recently, Donati et al. (1992) (see also these proceedings) obtained the first magnetic image of a cool star other than the Sun (V711 Tau). At one epoch they found a ring of clockwise field ( $\approx 300 \mathrm{G}$ ) surrounding a polar spot, while a region of counterclockwise field $(\approx-700 \mathrm{G})$ resided near an equatorial cool spot.

For the Sun we can measure plage coverages of up to $5 \%$ of the surface of the visible hemisphere. If we consider the most active stars one finds $\mathrm{Ca}$ II $\mathrm{H}$ and $\mathrm{K}$ emission higher by a factor 10 or more compared to solar-type stars. Assuming solar analogy it follows that about $65 \%$ of a stellar surface would be covered with plages (Oranje 1983). So far, AR Lac is the only star were plages were spatially resolved and Neff et al. (1989) obtained a plage coverage of $17 \%$ (additional to a large uniform component from the network). Still, this seems to be significantly smaller than the $65 \%$ cited by Oranje based on an extrapolated solar analogy and the $50 \%$ magnetic field filling factor on V833 Tau determined by Saar. Again the lesson seems clear: analogies to solar activity tracers are successful in explaining stellar observations qualitatively but simple scaling does not represent the observations quantitatively.

\section{OBSERVATIONAL EVIDENCE FOR DIFFERENTIAL ROTATION}

The solar case: from integrated sunlight

The detection of differential rotation from integrated sunlight has a long history and I am aware of only one positive detection: Keil 1990 (priv communication to Bruning 1991) from CaII K-line measurements carried out on a daily basis 
for over seven years! All other searches had no positive detection (see the introduction of Bruning 1991). The main reason being the time evolution of active regions and their random appearance in longitude (LaBonte 1982). This sets the stage for stellar measurements.

Stars: from rotational modulation of disk integrated flux

A particular photometric period can be interpreted as the rotation period of the star at the latitude of a particular spot. If we observe different periods at different times one may determine the amount of differential rotation. Extensive data base on stellar rotation periods from rotational modulation exist now, e.g. from $\mathrm{H}$ and $\mathrm{K}$ observations at Mt. Wilson (Baliunas et al. 1983, Duncan et al. 1991 ) and from broad-band photometry with robotic telescopes (Strassmeier et al. 1989, Hooten \& Hall 1990, Boyd et al. 1990). An alternative explanation for the observed period variations was proposed by Lanza et al. (1992) based upon the findings of Zappala \& Zuccarello (1989) that the rotational angular velocity of young sunspot groups is on average significantly higher than for groups older than one solar rotation. Present stellar data however cannot discern between the two possibilities.

However, there is a major problem with all of these long-term studies of rotational modulation. One must assume that the lifetime of a particular activity tracer is longer, or at least comparable, to the time scale of the studied phenomenon. To detect differential rotation, for example, one must make sure to exclude evolutionary effects of individual active regions. Hall (1991a) and Hall \& Busby (1990) investigated differential rotation from long-term starspot photometry. They found several stars that show evidence of differential surface rotation. Among those not listed by Lanza et al. (1992) are V478 Lyr, HR 7428, HD 37824 (Hall 1991a), but also BY Dra (Oskanyan et al. 1977), $\lambda$ And (Scaltriti et al. 1984), II Peg (Bohusz \& Udalski 1981), SV Cam (Zeilik et al. 1988), EI Eri (Strassmeier 1990), a.o.. But to be honest, none of these determinations is really conclusive. Moreover, there are several nondetections despite rather extended time coverages, e.g. FK Com (Jetsu et al. 1991), HD 199178 (Jetsu et al. 1990), VY Ari (Strassmeier \& Bopp 1992). Nevertheless it seems well established now that, if solar-type differential rotation exists on other stars, it is smaller by a factor of ten as compared to the Sun.

Baliunas et al. (1985) likely detected differential rotation on four solar-type stars monitored by the Mt. Wilson $\mathrm{H}$ and $\mathrm{K}$ photometer: HD 206860, HD 101501, HD 190406 , and HD 114710 ( $=\beta \mathrm{Com}$ ), while for eight other stars possible detections were reported. Recently, Donahue \& Baliunas (1992) presented further data on $\beta$ Com (G0V) and found strengthened evidence for differential rotation from a secular change of the seasonal rotation periods (Fig. 4; adapted from Donahue \& Baliunas). They suggest that the star may have two latitudinal zones of activity. One in which the rotation periods appear to follow a Ca II $\mathrm{H}$ and $\mathrm{K}$ activity cycle, and one that does not. The equator-to-pole variation in the solar rotation is $\approx$ $30 \%$, compared to 5 through $21 \%$ for the above stars ( $21 \%$ for $\beta$ Com), thus smaller than on the Sun. However, LaBonte (1984) and Baliunas et al. (1985) 
point out that, for the Sun, the expected range of rotation-period variations would be approximately a factor ten smaller if the Sun would be observed like a star. This is because the differential rotation tracers on the Sun appear primarily at low latitudes. Bruning (1991) nicely demonstrated the visibility of differential rotation from solar mean magnetic field measurements and from a simulated stellar data set. He concluded that (1) a large number of gaps in the data coverage prevents the detection, (2) the amount of differential rotation will be underestimated because, in general, a limited range of stellar latitudes will be observed and (3) without detailed surface mapping of the active regions one cannot distinguish between pole-to-equator and equator-to-pole migration.

Stars: from surface imaging

Vogt \& Hatzes (1991) found that the primary of UX Ari rotates differentially in the sense opposite to the Sun, i.e. zones near the pole of UX Ari rotate faster than zones near the equator. From three Doppler maps obtained within a five-month interval they identified two persistent features close to the (visible) pole and two near the equator. Measuring the relative rotation rates for these features enabled them to derive a differential rotation rate which is ten times smaller than the solar value (Fig. 5; adapted from Vogt \& Hatzes). This is in excellent agreement with results from long-term photometry (Rodonó 1986, Hall 1991a). Although this detection is probably the most convincing of all, there is some debate whether Doppler imaging reliably recovers surface detail at or near the rotation pole (cf. Strassmeier et al. 1991, Donati 1992, these proceedings, but see also Fig. 6a,b). Figure 6 is a new Doppler map of LQ Hya obtained from 9 different lines and does not show a polar cap-like spot but still has a feature at very high latitude.

\section{What about internal differential rotation?}

The question probably should be "Is there any evidence for internal differential rotation from surface activity tracers?". Well, yes there is, but I'd call it very vague.

The single G8 giants HR 1362 and HD 181943 (see Strassmeier et al. 1990b; Hooten \& Hall 1990 and Strassmeier 1991, respectively) are the two sole examples, at least to my knowledge, of stars with strong $\mathrm{Ca} I \mathrm{H}$ and $\mathrm{K}$ emission and very slow rotation ( $P_{\text {phtm }}=335$ days, and 385 days, respectively). Their chromospheric activity levels are one to two orders of magnitude higher than expected from the empirical activity-rotation relations for giants (e.g. Strassmeier et al. 1990a; Fig. 7). Either something has spun them down while maintaining the magnetic flux, or the photometric periods are not the rotation periods and the stars are viewed at very low inclinations (if so one needs to explain the fairly large photometric amplitudes). Both stars still have substantial lithium on their surface. From an evolutionary viewpoint, they are likely in a stage of internal radial redistribution of mass caused by the rapid decrease of the thickness of the $\mathrm{H}$-burning shell. Evolutionary models of cool giants normally assume rigid-body rotation (e.g. Rutten \& Pylyser 1988), but see also Endal \& Sofia (1979) and Tas- 
soul \& Tassoul (1989), and the new rotating stellar evolution models of the Sun (Pinsonneault et al. 1989). Durney \& Robinson (1982) predict a simple dynamo theory that relates surface activity to angular rotation and depth-dependent differential rotation. Thus, for the case of HR 1362 and HD 181943, one may argue that the period at the layer where the dynamo is believed to function ( $a$ thin layer at the bottom of the convection zone, Parker 1975), not the surface period, determines the activity level.

Observations of stellar rotation rates in open clusters of various age (cf. Hartmann \& Noyes 1987) indicate that solar-type stars reach their maximum rotation at an age of $\approx 10^{7-8}$ years and undergo rapid spin-down therafter. This rapid spin-down may cause large internal differential rotation between the radiative interior and the convective envelope, with the interior rotating more rapidly than the outside. The evolutionary models of Endal \& Sofia (1981) predict approximately a factor of 5 to 10 (at most) for the difference between the rotation periods at the surface and at the radiative-convective interface.

There is some observational evidence that evolved active stars with large spots also show enhanced surface lithium abundances (Fekel 1988, Pallavicini et al. 1992). This is somewhat of a puzzle because lithium should be well mixed by convection and destroyed during their (hotter) main-sequence lifetime. Fekel \& Balachandran (1992) suggest a scenario in which the surface-convection zone reaches a rapidly rotating core as the star evolves along the giant branch and dredges up to the surface high angular momentum material and freshly synthesized lithium. The rotating stellar evolution models of Pinsonneault et al. (1989) predict a rapidly rotating core and a spun-down convective layer for the Sun. It seems that the existence of $\mathrm{Li}$ rich giants is in agreement with theory. But what about spotted stars? Could it be that the large spotted areas of up to $20 \%$ of the entire stellar surface (e.g. HD 12545, Strassmeier \& Olah 1992) are regions of enhanced convection where most of the freshly synthesized lithium is dredged up? This could be checked by observations of rotational modulation of the lithium line.

Further evidence for the existence of internal differential rotation in binary star components comes from the connection between long-term brightness variations and orbital period changes of the eclipsing binary CG Cyg (Hall 1991b). Applegate (1992) predicts a large quadrupole moment by an alteration of the internal differential rotation gradient to predict that outer layers of a component in a binary star should rotate a little faster. According to this theory maximum luminosity should coincide with an O-C curve minimum if the star's outside spins faster than the inside but should coincide with an $\mathrm{O}-\mathrm{C}$ maximum if the reverse is true. For CG Cyg, a period increase is clearly observed (Hall 1991b; Fig. 8) and occurs at maximum light, thus the sense of differential rotation is that the outside rotates faster than the inside, according to Applegate's theory. 


\section{LOCAL VELOCITY FIELDS}

Photosphere

Granules are the most well-known tracers of local photospheric velocity fields but there are also larger scale velocity fields ("supergranulation"). Reviews of stellar granulation observations can be found in Gray (1988) and also in Dravins (1987). Line-bisector variations in phase with the rotation period were found on the G8 dwarf $\xi$ Boo A (Toner \& Gray 1988). This has been attributed to a surface feature carried across the stellar disk by rotation with a vertical velocity field enhanced over that of the rest of the surface. Toner \& LaBonte (1990) investigated the possibility that these line bisector variations may be caused by a mass flow tangential to the surface ("moat flow"). The latter model produces an equally good fit to the observations, and clearly more observations are needed to decide between the two hypotheses.

Note that the presence of starspots with the same velocity dispersion as the surrounding photosphere will also produce a line asymmetry probably best seen in correlative analysis between a spotted star and an inactive reference star (cf. Dempsey et al. 1992). This is in agreement with the finding of Bruning \& Saar (1990) that stronger asymmetry is observed for faster rotators.

\section{Chromosphere}

Neff (1992) describes a systematic redshift of $10 \mathrm{~km} \mathrm{~s}^{-1}$ of the observed $\mathrm{Mg}$ II line profiles from EI Eri, which could be either instrumental, or due to flows on a global scale in the stellar chromosphere. A similar redshift may also exist in the HD 199178 data. Crivellari et al. (1987) demonstrated the use of CaII $\mathrm{H}$-line profiles as a velocity field indicator. Strassmeier et al. (1992) monitored $\mathrm{H} \alpha$ line profile asymmetries of the young, active K star LQ Hya. Although the physical process of formation of these two lines is different, they contain similar information. In the case of LQ Hya there exists some evidence that velocity fields at the $\mathrm{H} \alpha$-forming layers are spatially related to the surrounding of photospheric spots. This is reminiscent of the observed mass outflow around sunspots (cf. Brickhouse \& LaBonte 1988). For IM Peg, Huenemoerder et al. (1990) noted that the hydrogen emission is not correlated with the starspots, but rather arises from the network. In UX Ari, however, Huenemoerder et al. (1989) detected modulation of chromospheric emission lines slightly preceeding the photometric minimum, indicating that the chromospheric emission originates close to, but outside of photospheric spots.

\section{Corona and the stellar environment}

Models of the quiescent solar corona are based on magnetic loops (Rosner et al. 1978). Solar observations confirm this picture and also detected systematic plasma flows. Vilhu (1987) and Vilhu \& Walter (1987) showed that there is an upper limit to the $\mathrm{x}$-ray flux, which suggests that saturation occurs when the loops fill the available coronal volume. First observational evidence for such extended geometries came from the detection of an x-ray eclipse of AR Lac. New ROSAT data will certainly expand our view of stellar coronae and their 
flow structure.

Several reviews on the subject of stellar flares can be found in IAU Colloquium 104 (Haisch \& Rodono 1989 and Haisch et al. 1991). Flares in RS CVn binaries were recently reviewed by Catalano (1990). For this section I pick out just a few highlights relating to the general topic of activity tracers for stars on the main-sequence stars or close to it. A particularly interesting discovery was the detection of a strong stellar wind from the K-dwarf/WD pair V471 Tau (Mullan et al. 1989), probably driven by sporadic coronal mass ejection. In another context, Houdebine et al. (1990) presented evidence of a high-velocity mass ejection event $\left(v_{\tau} \approx 5800 \mathrm{~km} \mathrm{~s}^{-1}\right)$ on AD Leo just starting with the onset of a particularly violent flare. There is now strengthened evidence for the existence of external plasma around the K dwarf of V471 Tau (see Young et al. 1988, 1990). Young et al. detected transient $\mathrm{H} \alpha$ emission components and their radial velocities reveal unambiguously that the emission cannot arise from the surface of the star and they suggested that it arises from plasma held in a ring or a disk orbiting the $\mathrm{K}$ dwarf (not the white dwarf). They also noted that these emission components are greatly enhanced immediately following the detection of a flare on the "surface" of the $\mathrm{K}$ dwarf. This leads to an inference of causality and suggests that this "ring" is feed by coronal mass ejection. Similar observations of transient absorption features were reported by Collier-Cameron \& Robinson (1989) for AB Dor, a young K0 star. Modeling with a two-cloud structure at a distance between 3-4 stellar radii embedded in and corotating with a hot extended corona indicates the presence of closed magnetic loops to very great heights.

\section{WHAT'S NEXT?}

Considerable progress is being made towards concerted multiwavelength observations of activity tracers at all atmospheric layers. However, the exclusive circle of stars suitable for such techniques is indeed very small. Only objects with large-scale, I mean big, really big, spots or plages can be recognized and studied since one needs rotationally modulated indicators. At present there are about 300 spotted stars, to my knowledge. The second edition of the Chromospherically Active Binary Star (CABS) catalog (Strassmeier et al. 1988) will contain a little more than 200 close binaries with at least one active late-type stellar component. Some of these stars would be ideal targets for a space mission like PRISMA (see Catala, these proceedings) which is capable of simultaneous soft $\mathrm{x}$-ray observations, UV spectroscopy, and visual and near-infrared photometry.

\section{ACKNOWLEDGEMENTS}

This work has been supported in part by FWF grant P7993, and the University of Vienna. I wish to thank Jim Neff, Bill Wehlau, and Michel Breger for a critical reading of the manuscript. I would like to thank Werner W. Weiss and the Scientific Organizing Committee for inviting me to present this review and for putting together such a stimulating meeting and the LOC for their continuous support and daily struggles. Last but not least, many thanks to numerous colleagues for sending me their work prior to publication. 


\section{REFERENCES}

Andrews, A. D., et al. 1988, A\&A 204, 177.

Applegate, J. H., 1992, ApJ, submitted.

Appourchaux, T., et al. 1991, PRISMA Assessment Study, ESA SCI 5.

Baliunas, S. L. 1988, in Automatic Small Telescopes, eds. D. S. Hayes, R. M. Genet, Fairborn Press, Mesa, p. 83.

Baliunas, S. L., Dupree, A. K., 1982, ApJ 252, 668.

Baliunas, S. L., et al. 1983, ApJ 275, 752.

Baliunas, S. L., et al. 1985, ApJ 294, 310.

Baliunas, S. L., Vaughan, A. H., 1985, ARA\&A 23, 379.

Basri, G., Laurent, R., Walter, F. M., 1985, ApJ 298, 761.

Böhm-Vitense, E., 1992, AJ 103, 608.

Bohusz, E., Udalski, A., 1981, Acta Astr. 31, 185.

Boyd, L. J., et al. 1990, IAPPP 42, 44.

Brickhouse, N. S., LaBonte, B. J., 1988, SP 115, 43.

Bruning, D. H., 1991, PASP 103, 368.

Bruning, D. H., Saar, S. H., 1990, in $6^{\text {th }}$ Cambridge Workshop Cool Stars, Stellar Systems, and the Sun, ed. G. Wallerstein, Kluwer, Dordrecht, PASPC 9, 165.

Byrne, P. B., 1986, IBVS 2951.

Catala, C., 1988, A\&A 193, 222.

Catala, C., Czarny, J., Felenbok, P., Talavera, A., The, P. S., 1991, A\&A 244, 166.

Catalano, S. 1990, NATO-ASI, Active Close Binaries, ed. C. Manoglu, Kluwer-Dordr., p. 411.

Collier-Cameron, A., Robinson, R. D., 1989, MNRAS 236, 57.

Crivellari, L., Beckman, J. E., Foing, B. H., Vladilo, G., 1987, A\&A 174, 127.

Dempsey, R. C., et al. 1992, ApJ, in press.

Donahue, R. A., Baliunas, S. L., 1992, ApJL, submitted.

Donati, J.-F., Semel, M., del Toro Iniesta, J. C., 1990, A\&A 233, L17.

Donati, J.-F., et al. 1992, A\&A, in press.

Doyle, J. G., et al. 1988, A\&A 192, 275.

Doyle, J. G., et al. 1989, A\&A 223, 219.

Dravins, D., 1987, A\&A 172, 200.

Duncan, D. K., et al. 1991, ApJS 76, 383.

Durney, B. R., Robinson, R. D., 1982, ApJ 253, 290.

Eaton, J. A. 1992, in Armagh Obs. Colloq., Surface Inhomogeneities in Late-type Stars, ed. P. B. Byrne, D. J. Mullan, Springer, Berlin Heidelberg New York, p. 15.

Endal, A. S., Sofia, S., 1979, ApJ 232, 531.

Endal, A. S., Sofia, S., 1981, ApJ 243, 625.

Fekel, F. C., 1988, in A Decade of UV Astronomy with IUE, ed. E. Rolfe, ESA SP-281, p. 331.

Fekel, F. C., Balachandran, S., 1992, ApJ, submitted.

Finkenzeller, U., Mundt, R., 1984, A\&AS 55, 109.

Gray, D. F., 1988, Lectures in Spectral Line Analysis: the F, G, $K$ stars, The Publisher, Ontario.

Gray, D. F., 1991, in NATO-ASI, Angular Momentum Evolution of Young Stars, Kluwer, p. 183.

Haisch, B., Rodonó, M., 1989, (eds), LAU Colloq. 104, Solar and Stellar Flares, SP 121.

Haisch, B., Strong, K. T., Rodonó, M., 1991, ARA\&A 29, 275.

Hall, D. S., 1991a, in IAU Colloq. 130, The Sun and Cool Stars: activity, magnetism, dynamos, eds. I. Tuominen, D. Moss, G. Rüdiger, Springer, p. 353.

Hall, D. S., 1991b, ApJ 380, L85.

Hall, D. S., Busby, M. R. 1990, in NATO-ASI, Active Close Binaries, ed. C. Ibanoglu, Kluwer, Dordrecht, p. 377.

Hartmann, L. W., Noyes, R. W., 1987, ARA\&A 25, 271. 
Hatzes, A. P., 1991 a, MNRAS 248, 487.

Hatzes, A. P., 1991b, MNRAS 253, 89.

Hatzes, A. P., Penrod, G. D., Vogt, S. S., 1989, ApJ 341, 456.

Hooten, J. T., Hall, D. S., 1990, ApJS 74, 225.

Houdebine, E. R., Foing, B. H., Rodonó, M., 1990, A\& A 238, 249.

Huenemoerder, D. P., Buzasi, D. L., Ramsey, L. W., 1989, AJ 98, 1398.

Huenemoerder, D. P., Ramsey, L. W., Buzasi, D. L., 1990, ApJ 350, 763.

Jetsu, L., Huovelin, J., Tuominen, I., Vilhu, O., Bopp, B. W., Piirola, V. 1990, A\&A, 236, 423.

Jetsu, L., Pelt, J., Tuominen, I., Nations, H. 1991, in IAU Colloq. 130, The Sun and Cool Stars: activity, magnetism, dynamos, eds. I. Tuominen, D. Moss, G. Rüdiger, Springer, p. 381.

LaBonte, B. J., 1982, ApJ 260, 647.

LaBonte, B. J., 1984, ApJ 276, 335.

Landstreet, J. D., 1991, in IAU Colloq. 130, The Sun and Cool Stars: activity, magnetism, dynamos, eds. I. Tuominen, D. Moss, G. Rüdiger, Springer, p. 342.

Lanza, A. F., Rodonó, M., Zappala, R. A., 1992, in Armagh Obs. Colloq., Surface Inhomogeneities in Late-type Stars, ed. P. B. Byrne, D. J. Mullan, Springer, p. 298.

Liebert, J., Probst, R. G., 1987, ARA\&A 25, 473.

Linsky, J. L., 1992, in Armagh Obs. Colloq., Surface Inhomogeneities in Late-type Stars, ed. P. B. Byrne, D. J. Mullan, Springer, Berlin Heidelberg New York, p. 113.

Marstad, N., et al. 1982, in Adv. in UV Astronomy: Four Years of IUE Research, NASA Conf. Publ. 2238, p. 554.

Mathys, G., Solanki, S. K., 1989, A\&A 208, 189.

Mattig, W., Kneer, F., 1978, A\&A 65, 11.

Mullan, D. J., Sion, E. M., Bruhweiler, F. C., Carpenter, K. G., 1989, ApJ 339, L33.

Neff, J. E., 1991, in IAU Colloq. 130, The Sun and Cool Stars: activity, magnetism, dynamos, eds. I. Tuominen, D. Moss, G. Rüdiger, Springer, Berlin Heidelberg New York, p. 330.

Neff, J. E., 1992, in Armagh Obs. Colloq., Surface Inhomogeneities in Late-type Stars, ed. P. B. Byrne, D. J. Mullan, Springer, p. 54.

Neff, J. E., Walter, F. M., Rodonó, M., Linsky, J. L., 1989, A\&A 215, 79.

Olah, K., Hall, D. S., Henry, G. W., 1991, A\&A 251, 531.

Olah, K., Pettersen, B. R., 1991, A\&A 242, 443.

Oranje, B. J., 1983, A\&A 124, 43.

Oskanyan, V. S., Evans, D. S., Lacy, C., McMillan, R. S., 1977, ApJ 214, 430.

Pagano, I., Rodono, M., Neff, J. E., 1992, in Armagh Obs. Colloq., Surface Inhomogeneities in Late-type Stars, ed. P. B. Byrne, D. J. Mullan, Springer, p. 315.

Pallavicini, R., 1992, in NATO-ASI, The Sun: a laboratory for Astrophysics, J. Brown and J. Schmelz (eds.), in press.

Pallavicini, R., Randich, S., Giampapa, M., 1992, A\&A 253, 185.

Parker, E. N., 1975, ApJ 198, 205.

Pasquini, L., Brocato, E., Pallavicini, R., 1990, A\&A 234, 277.

Pasquini, L., Pallavicini, R., Pakull, M., 1988, A\&A 191, 253.

Pinsonneault, M. H., Kawaler, S. D., Sofia, S., Demarque, P., 1989, ApJ 338, 424.

Praderie, F., Simon, T., Catala, C., Boesgaard, A. M., 1986, ApJ 303, 311.

Ramsey, L. 1990, in $6^{\text {th }}$ Cambridge Workshop Cool Stars, Stellar Syotems, and the Sun, ed. G. Wallerstein, Kluwer, Dordrecht, PASPC 9, 195.

Rice, J. B., 1991, A\&A 245, 561.

Rice, J. B., Wehlau, W. H., 1990, A\&A 233, 503.

Rice, J. B., Wehlau, W. H., 1991, A\&A 246, 195.

Rodonó, M., 1986, in $4^{\text {th }}$ Cambridge Workshop Cool Stars, Stellar Systems, and the Sun, eds. M. Zeilik, D. M. Gibson, Springer, p. 475.

Rodonó, M. et al. 1987, A\&A 176, 267. 
Rosner, R., Tucker, W. H., Vaiana, G. S., 1978, ApJ 220, 643.

Rutten, R. G. M., Pylyser, E., 1988, A\&A 191, 227.

Saar, S. H., 1988, ApJ 324, 441.

Saar, S. H., 1991, in IAU Colloq. 130, The Sun and Cool Stors: activity, magnetism, dynamos, eds. I. Tuominen, D. Moss, G. Rüdiger, Springer, p. 389.

Saar, S. H., Neff, J. E., 1990, in $6^{\text {th }}$ Cambridge Workshop Cool Stars, Stellar Systems, and the Sun, ed. G. Wallerstein, Kluwer, Dordrecht, PASPC 9, 171.

Scaltriti, F., et al. 1984, A\&A 139, 25.

Schmidt, H. U., 1992, in Cowling Memorial Symp. Solar System Plasma Physics, Geophys. Astrophys. Fluid Dyn., in press.

Schrijver, C. J., 1987, A\&A 172, 111.

Schrijver, C. J., 1990, A\&A 234, 315.

Shine, R. A., Linsky, J. L., 1972, SP 25, 357.

Simon, T., Fekel, F. C., 1987, ApJ 316, 434.

Simon, T., Landsmann, W., 1991, ApJ 380, 200.

Stepien, K., 1989, Acta Astr. 39, 209.

Strassmeier, K. G. 1990, ApJ 348, 682.

Strassmeier, K. G., 1991, IBVS 3618.

Strassmeier, K. G., 1992, in Proc. $103^{\text {rd }}$ ASP Meeting, Robotic Telescopes for the 1990 's, ed. A. Filippenko, PASPC, in press.

Strassmeier, K. G., Bopp, B. W. 1992, A\&A, in press.

Strassmeier, K. G., Hall, D. S., Boyd, L. J., Genet, R. M. 1989, ApJS 69, 141.

Strassmeier, K. G., Hall, D. S., Zeilik, M., Nelson, E., Eker, Z., Fekel, F. C. 1988, A\&AS 72, 291.

Strassmeier, K. G., Olah K., 1992, A\&A, in press.

Strassmeier, K. G., et al. 1990a, ApJS 72, 191.

Strassmejer, K. G., et al. $1990 \mathrm{~b}, \mathrm{ApJ} 350,367$.

Strassmeier, K. G., et al. 1991, A\&A 247, 130.

Strassmeier, K. G., Rice, J. B., Wehlau, W. H., Hill, G., M., Matthews, J. M., 1992, A\&A, submitted.

Tassoul, J.-L., Tassoul, M., 1989, A\&A 213, 397.

Tokovinin, A. A., 1990, SvA Lett. 16, 1026.

Toner, C. G., Gray, D. F., 1988, ApJ 334, 1008.

Toner, C. G., LaBonte, B. J., 1990, in $6^{\text {th }}$ Cambridge Workshop Cool Stars, Stellar Systems, and the Sun, ed. G. Wallerstein, Kluwer, Dordrecht, PASPC 9, 161.

Vilhu, O., 1990, in Proc. Nordic-Baltic Astronomy Meeting, eds. C.-I. Lagerkvist et al., Uppsala, Repr. HSC, p. 369.

Vilhu, O., Gustafsson, B., Walter, F. M., 1991, A\&A 241, 167.

Vogt, S. S. 1988, in IAU Symp. 132, The Impact of Very High $S / N$ Spectroscopy on Stellat Physics, eds. G. Cayrel de Strobel, M. Spite, Kluwer, Dordrecht, p. 253.

Vogt, S. S., Hatzes, A. P. 1991, in IAU Colloq. 130, The Sun and Cool Stars: activity, magnetism, dynamos, eds. I. Tuominen, D. Moss, G. Rüdiger, Springer, p. 297.

Walter, F. M., Gibson, D. M., Basri, G., 1983, ApJ 267, 665.

Walter, F. M., et al. 1987, A\&A 186, 241.

Wilson, O. C., 1978, ApJ 226, 379.

Wolf, S. C., Boesgaard, A. M., Simon, T., 1986, ApJ 310, 360.

Young, A., Ajir, F., Thurman, G., 1989, PASP 101, 1017.

Young, A., Skumanich, A., Paylor, V., 1988, ApJ 334, 397.

Young, A., Rottler, L., Skumanich, A., 1991, ApJ 378, L25.

Zappala, R. A., Zuccarello, F., 1989, A\&A 214, 369.

Zeilik, M., De Blasi, C., Rhodes, M., Budding, E. 1988, ApJ 332, 293.

Zirin, H., 1988, Astrophysics of the Sun, Cambridge Univ. Press, Cambridge. 


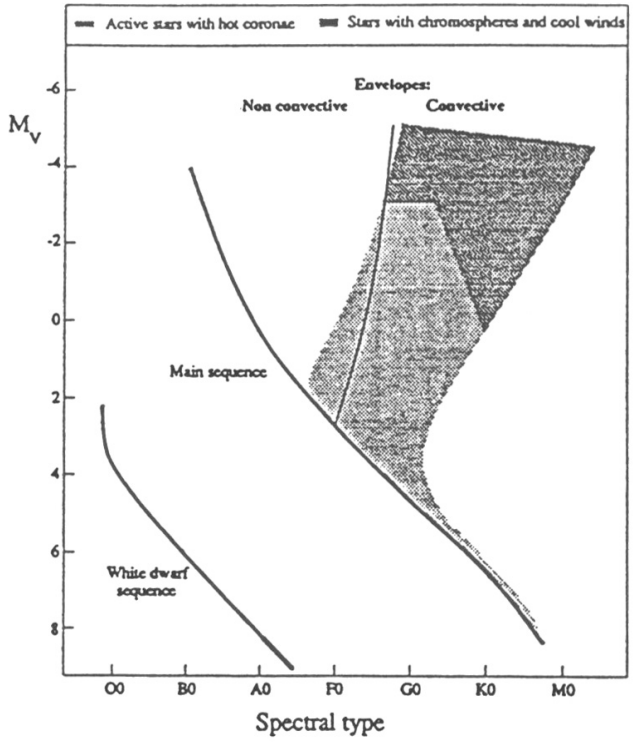

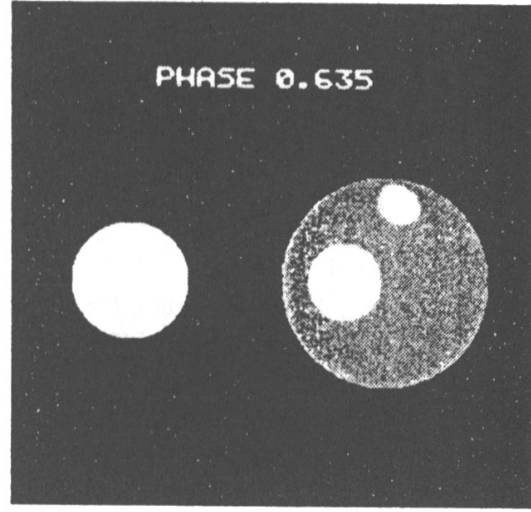

Fig. 2

Fig. 1

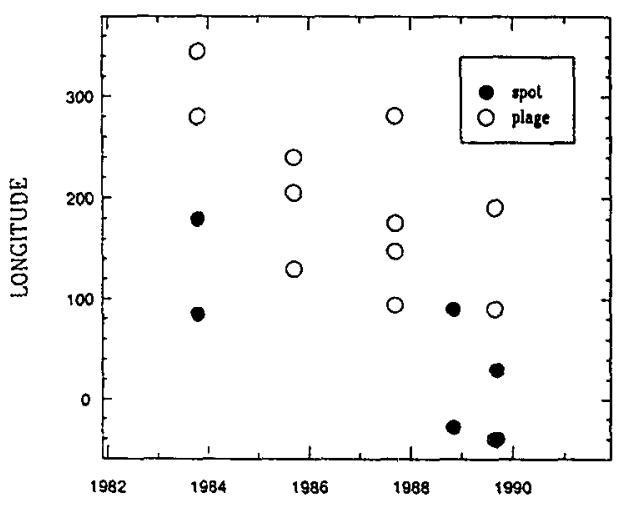

Fig. 3

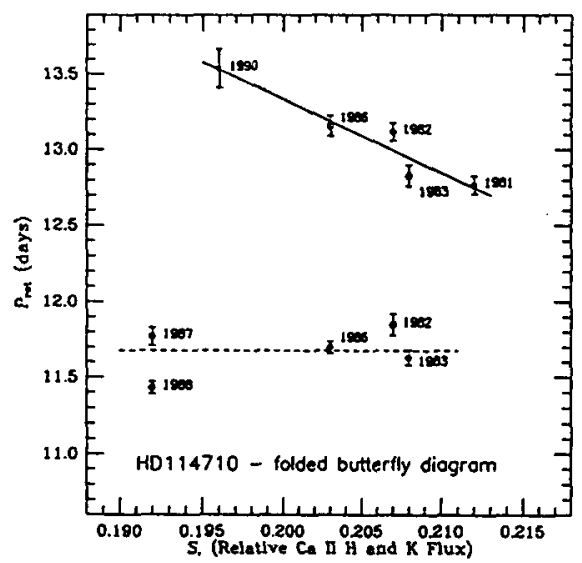

Fig. 4

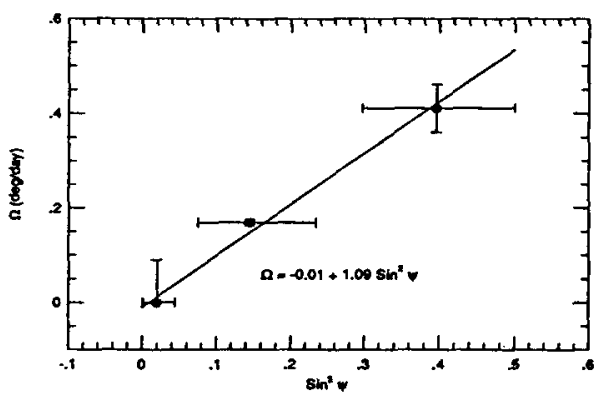

Fig. 5 

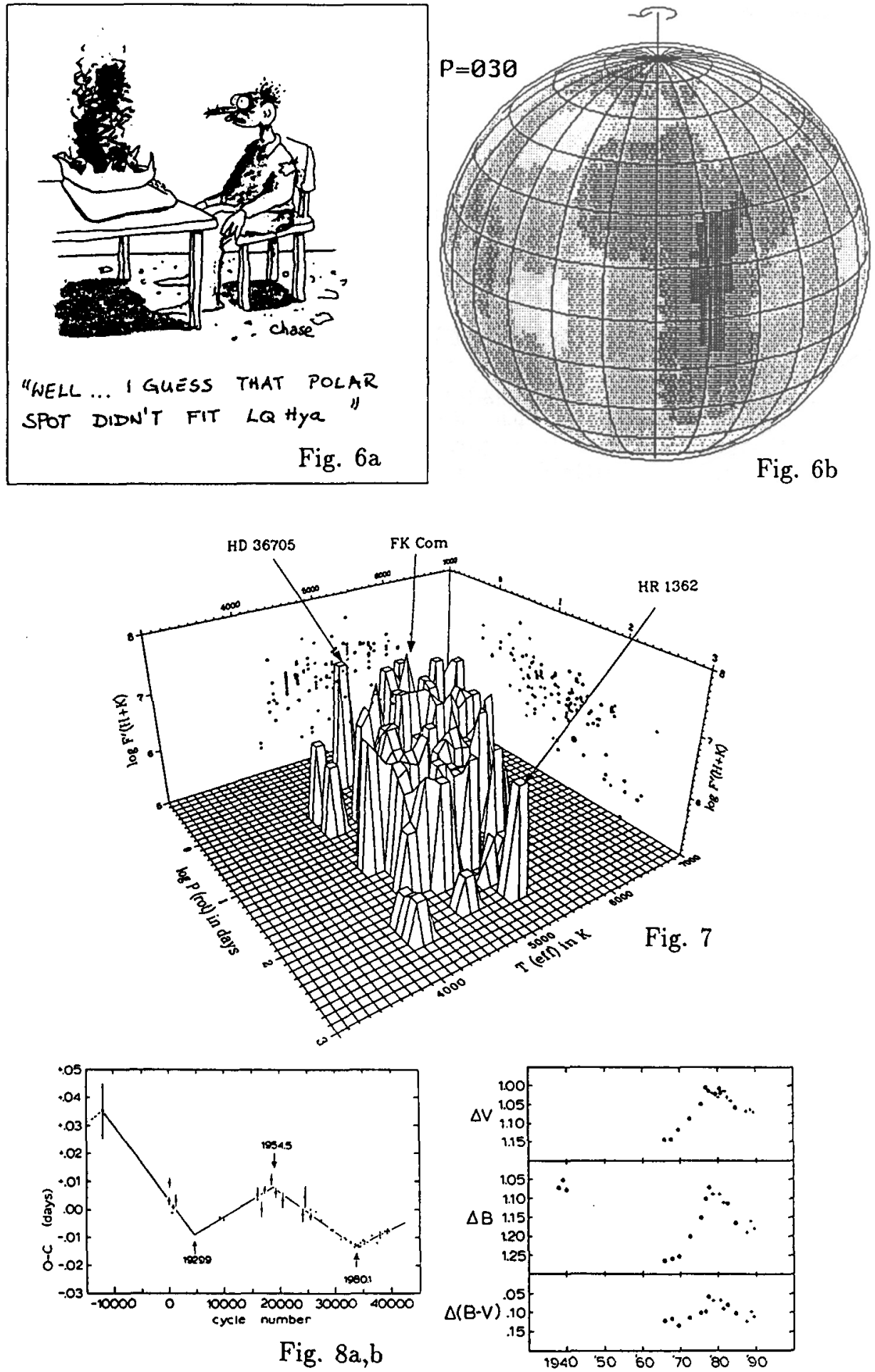